\title{
Adaptive optics sky coverage modeling for extremely large telescopes
}

\author{
Richard M. Clare, Brent L. Ellerbroek, Glen Herriot, and Jean-Pierre Véran
}

\begin{abstract}
A Monte Carlo sky coverage model for laser guide star adaptive optics systems was proposed by Clare and Ellerbroek [J. Opt. Soc. Am. A 23, 418 (2006)]. We refine the model to include (i) natural guide star (NGS) statistics using published star count models, (ii) noise on the NGS measurements, (iii) the effect of telescope wind shake, (iv) a model for how the Strehl and hence NGS wavefront sensor measurement noise varies across the field, (v) the focus error due to imperfectly tracking the range to the sodium layer, (vi) the mechanical bandwidths of the tip-tilt (TT) stage and deformable mirror actuators, and (vii) temporal filtering of the NGS measurements to balance errors due to noise and servo lag. From this model, we are able to generate a TT error budget for the Thirty Meter Telescope facility narrow-field infrared adaptive optics system (NFIRAOS) and perform several design trade studies. With the current NFIRAOS design, the median TT error at the galactic pole with median seeing is calculated to be $65 \mathrm{~nm}$ or 1.8 mas rms. (C) 2006 Optical Society of America

OCIS codes: $\quad 010.1080,010.7350$.
\end{abstract}

\section{Introduction}

Several extremely large telescopes (ELTs) of primary mirror diameters 20-100 $\mathrm{m}$ are currently in the planning and design stages, such as the Thirty Meter Telescope (TMT), ${ }^{1}$ the European ELT, ${ }^{2}$ the Giant Magellan Telescope (GMT), ${ }^{3}$ and the Euro50 project. ${ }^{4}$ Successful utilization of the unprecedented resolution of these ELTs is contingent upon the correction of atmospheric turbulence with adaptive optics (AO). ${ }^{5}$

Current AO systems ${ }^{6}$ employ a laser guide star (LGS) to provide wavefront sensor (WFS) measurements of the instantaneous wavefront aberrations without relying upon the availability of a bright natural guide star (NGS). However, because the laser jitters and is deflected on both the upward and downward paths through the atmosphere, the tip-tilt (TT) modes of the atmospheric aberration cannot be determined from the LGS. Consequently, NGS WFS(s) are also required to estimate these modes. The sky coverage problem is the probability of finding suffi-

R. M. Clare (rclare@keck.hawaii.edu) and B. L. Ellerbroek are with the Thirty Meter Telescope Project, California Institute of Technology, 1200 E. California Boulevard, Mail Code 102-8, Pasadena, California 91125. G. Herriot and J.-P. Véran are with the Herzberg Institute of Astrophysics, 5071 West Saanich Road, Victoria, British Columbia, Canada.

Received 12 May 2006; revised 4 August 2006; accepted $11 \mathrm{Au}-$ gust 2006; posted 11 August 2006 (Doc. ID 70899).

0003-6935/06/358964-15\$15.00/0

(C) 2006 Optical Society of America ciently bright NGS(s) within the isoplanatic patch of the science object that will allow for the accurate estimation of the TT modes.

Multiconjugate adaptive optics (MCAO), where there are multiple deformable mirrors (DMs) conjugate to different altitudes in the atmosphere, have been proposed to overcome the cone effect and provide imaging over a wider field of view (FOV). Multiple LGSs are used to tomographically reconstruct atmospheric turbulence in three dimensions and determine the commands for the multiple deformable mirrors. An alternative $\mathrm{AO}$ configuration for tomography and wide-field correction is multiobject adaptive optics (MOAO), where the DMs apply independent corrections to objects within the FOV.

An additional fundamental effect limiting LGS AO systems is tilt anisoplanatism, which arises when a single TT NGS is viewed off axis with respect to the science object. For LGS MCAO, tilt anisoplanatism will degrade the uniformity of turbulence compensation over an extended field and may become the dominant wavefront error term. A number of different approaches to overcome tilt anisoplanatism have been proposed: (1) using multiple TT NGSs, ${ }^{7}$ (2) using a NGS that measures TT and focus, ${ }^{8}$ and (3) using LGSs at different altitudes ${ }^{7-9}$ (i.e., a combination of Rayleigh and sodium LGS). For option (1), Ellerbroek and Rigaut ${ }^{7}$ state that only three TT NGSs are necessary, and we use three NGS WFSs for this option. Because the altitude of the sodium layer is constantly changing, ${ }^{10}$ and it is not possible to disentangle at- 
mospheric focus aberrations from these altitude variations with the LGS measurements, we modify option (1) to be two TT sensors and a TT-focus sensor. In this paper, we evaluate the performance of these three options.

In Ref. 11, a method for producing Monte Carlo sky coverage simulations over random NGS constellations is presented. The essence of this method is to propagate the turbulence phase screens at each altitude, which are represented as a Zernike basis sum, to the aperture using geometric optics. This model accounts for the cone effect for the finite height of the LGSs as well as the anisoplanatism caused by the guide stars being off axis with respect to the science object. The expected wavefront error is then calculated using a minimum variance estimator from these transformation matrices and the statistical properties of the atmosphere.

In this paper, we continue with the methodology set out in Ref. 11 but include a number of practical considerations in order to generate a TT error budget for the narrow-field infrared adaptive optics system (NFIRAOS) of the TMT. ${ }^{12}$ These upgrades include (i) guide star statistics using the Bahcall-Soneira and Spagna models; (ii) background, photon, and readout noise on the NGS measurements; (iii) a telescope windshake model; (iv) a model for how the Strehl, and hence the NGS WFS measurement noise, varies across the field in the IR; (v) the error due to imperfectly tracking the range to the sodium layer; (vi) the mechanical bandwidths of the NFIRAOS TT stage and DM actuators; and (vii) temporal filtering of the NGS measurements to balance the errors due to wind shake, noise, servo lag, and sodium altitude tracking. We employ this updated sky coverage model to perform a number of design trades for the NFIRAOS. These include whether to use optical ( $V$ band) or IR ( $J$ band) stars and sensors for the NGS WFS. Second, we find the optimal patrol field diameter required to find sufficient NGS. Third, we use this methodology to evaluate the different methods for correcting the tilt anisoplanatism described previously. We also compare the performance of a quad-cell Shack-Hartmann (SH) detector with a matched-filter or noise-weighted leastsquares approach for measuring the TT from the NGS.

The theory of the sky coverage simulator, including the transformation matrices, control algorithm, and minimum variance reconstructors, is presented in Section 2. The computational details of the sky coverage simulator, in particular, the calculation of the noise and atmospheric covariance matrices, are outlined in Section 3. Sky coverage Monte Carlo simulation results for NFIRAOS are presented in Section 4. Conclusions are drawn in Section 5.

\section{Theory}

\section{A. Background and Notation}

We consider the atmosphere to consist of $N_{l}$ discrete layers of turbulence. We model the phase screen at the $i$ th atmospheric layer, $\phi(i ; r, \theta)$, as a finite sum of
$N$ orders of Zernike polynomials ${ }^{13}$ :

$$
\phi(i ; r, \theta)=\sum_{n=0}^{N} \sum_{m=0}^{n} a_{n m}(i) Z_{n m}(r, \theta),
$$

where $Z_{n m}$ is the $m$ th Zernike polynomial of radial order $n$, and $a_{n m}(i)$ are the coefficients of the corresponding Zernike polynomials at the $i$ th layer.

For each WFS in the AO system, the phase screens, represented by a vector of Zernike coefficients at each atmospheric layer, $a$, are projected to the aperture plane by a transformation matrix, $T$, such that

$$
b=T a,
$$

where $b$ is the vector of the Zernike coefficients of the wavefront measured by the WFS, and

$$
a=\left[\begin{array}{lllll}
a(1) & \cdots & a(i) & \cdots & a\left(N_{l}\right)
\end{array}\right]^{T} .
$$

The $T$ matrix takes into account the off-axis effect of the NGS or LGS, as well as the cone effect due to the finite height of the LGS, and also includes a summation over all of the atmospheric layers. The exact form of the $T$ matrix is defined in Ref. 11. The wavefront modes as seen by the LGS, $b_{L}$ (both sodium, and when applicable, Rayleigh), are given by

$$
b_{L}=T_{L} a,
$$

where the $L$ subscript in this paper refers to the LGS. In this paper, the LGS measurements are assumed to be noiseless and instantaneous since, we are primarily concerned with the dynamic errors with respect to the NGS, and only the lower-order component of the TT-removed LGS measurements are considered (typically Zernike radial orders 2-6). The effects of noise on the LGS for the TMT are addressed in Ref. 14 .

Similarly, the wavefront modes seen from the NGS, $b_{n}$, are given by

$$
b_{n}=T_{n} a^{\prime}+n^{\prime},
$$

where the $n$ subscript refers to the NGS, and the vector $n^{\prime}$ is the additive noise on the NGS WFS measurements as discussed further in Subsection 3.D. The prime (') notation on $a$ and $n$ is to show that the NGS measurements, $b_{n}$, are temporally filtered by a controller $H_{\mathrm{ol}}(s)$ to minimize the combined effect of the measurement noise and the servo lag.

The Zernike coefficients, $b_{s}$, of the wavefronts projected into the aperture plane for sources in the directions of the science evaluation points are given by

$$
b_{s}=T_{s} a
$$


where the $s$ subscript refers to the science field. The $N_{s}$ science evaluation points we consider for NFIRAOS are defined in Section 4.

Finally, the DMs provide a correction to the wavefronts associated with the science evaluation directions that is given by

$$
b_{m}=T_{m} P_{m} \hat{a},
$$

where $\hat{a}$ is the estimate of the Zernike coefficients at each layer; the $m$ subscript refers to mirrors throughout this paper; and $P_{m}$, the optimal fit of the Zernike modes to the DM to compensate for the estimated science phase profiles, is defined in Ref. 11 by

$$
P_{m}=\left(T_{m}{ }^{T} W T_{m}\right)^{-1} T_{m}{ }^{T} W T_{s} .
$$

Here, $W$ is a block diagonal weighting matrix of $N_{s}$ blocks whose elements are $w_{k} I$, where $I$ is an identity matrix of dimensions equal to the number of Zernike modes computed for each science wavefront. If the entire science field is considered, $w_{k}=1 / N_{s}$ for all $k$ points. If only the on-axis science point is considered, $w_{1}=1$ and $w_{k}=0$, otherwise. Similar mirror transformation matrices can be derived for the correction of the NGS modes, $T_{m, n}$, and the correction of the LGS modes, $T_{m, L}$.

\section{B. Control Algorithm Overview}

The control algorithm we employ in this paper is a two-step process. We first estimate the wavefront Zernike coefficients at each layer, $a$, and the associated NGS measurements, $b_{n}$, from the LGS measurements, $b_{L}$. The NGS WFS measurements are estimated by first performing atmospheric tomography to estimate the full $3 \mathrm{D}$ turbulence profile from the LGS WFS measurements and then projecting the volume turbulence estimate into wavefront estimates in the direction of each NGS. Of course, the overall TT mode of each NGS measurement will be poorly estimated due to the LGS position uncertainty. But reasonable estimates of the focus and astigmatism modes (as well as the tilt anisoplanatism modes) can be obtained and can be incorporated into the wavefront reconstruction process. We then estimate the residual uncertainty in $a, a_{\text {res }}$, from the new information provided by the actual NGS measurements, $b_{n}$. It may be shown that this two-step approach is equivalent to an integrated one-step minimum variance estimator, but the two-step approach is a more efficient approach to Monte Carlo simulations of the NGS constellations. The control model is shown in Fig. 1 and is described in more detail here.

First, the Zernike coefficients at each of the atmospheric layers are estimated from the LGS measurements, $b_{L}$, using

$$
\hat{a}_{L}=E_{L} b_{L}=E_{L} T_{L} a,
$$

where $E_{L}$ is a minimum variance estimator as described in Subsection 2.C. The residual error in this estimate is given by

$$
a_{\mathrm{res}}=a-\hat{a}_{L}
$$

and from Fig. 1 the estimate of the residual error (computed from the NGS measurements) is related to the final estimate $\hat{a}$ by

$$
\hat{a}_{\text {res }}=\hat{a}-\hat{a}_{L} .
$$

Note that although Eq. (9) indicates that the LGS WFS measurement vector $b_{L}$ is measured in an open loop without the corrections applied by the DMs, NFIRAOS will actually generate pseudo-open-loop measurements by combining closed-loop measurements with the knowledge of DM actuator commands, as illustrated in Fig. 1.

Next, the residual uncertainty, $a_{\text {res }}$, is estimated based upon the new information provided by the temporally filtered NGS WFS measurement. Temporal filtering is applied to balance the estimation errors due to NGS noise, servo lag, sodium-layer altitude uncertainty, and wind shake. The filtered NGS WFS measurement is given by

$$
\begin{aligned}
b_{n} & =\frac{H_{\mathrm{ol}}(s)}{1+H_{\mathrm{ol}}(s)}\left(T_{n} a+n\right) \\
& =H_{\mathrm{cl}}(s)\left(T_{n} a+n\right) \\
& =T_{n}\left[H_{\mathrm{cl}}(s) a\right]+H_{\mathrm{cl}}(s) n \\
& =T_{n} a^{\prime}+n^{\prime},
\end{aligned}
$$

where $H_{\mathrm{cl}}(s)$ is the closed-loop temporal filter, and $s$ is the Laplace coordinate. The new information provided by this measurement is the component not predicted by the LGS WFS measurement,

$$
\delta b_{n}=b_{n}-\hat{b}_{n}=b_{n}-E_{n} b_{L},
$$

where $E_{n}$ is the minimum variance estimator for $b_{n}$ as described further in Subsection 2.C. Combining Eqs. (12) and (13) yields

$$
\delta b_{n}=T_{n} a^{\prime}+n^{\prime}-E_{n} T_{L} a .
$$

Finally, the estimate of the residual uncertainty $\hat{a}_{\text {res }}$ is given by the expression

$$
\hat{a}_{\text {res }}=E_{\text {res }} \delta b_{n},
$$

where $E_{\text {res }}$ is a minimum variance estimator as described further in Subsection 2.C. The derivations of $E_{L}, E_{n}$, and $E_{\text {res }}$, and the associated formulas for eval- 


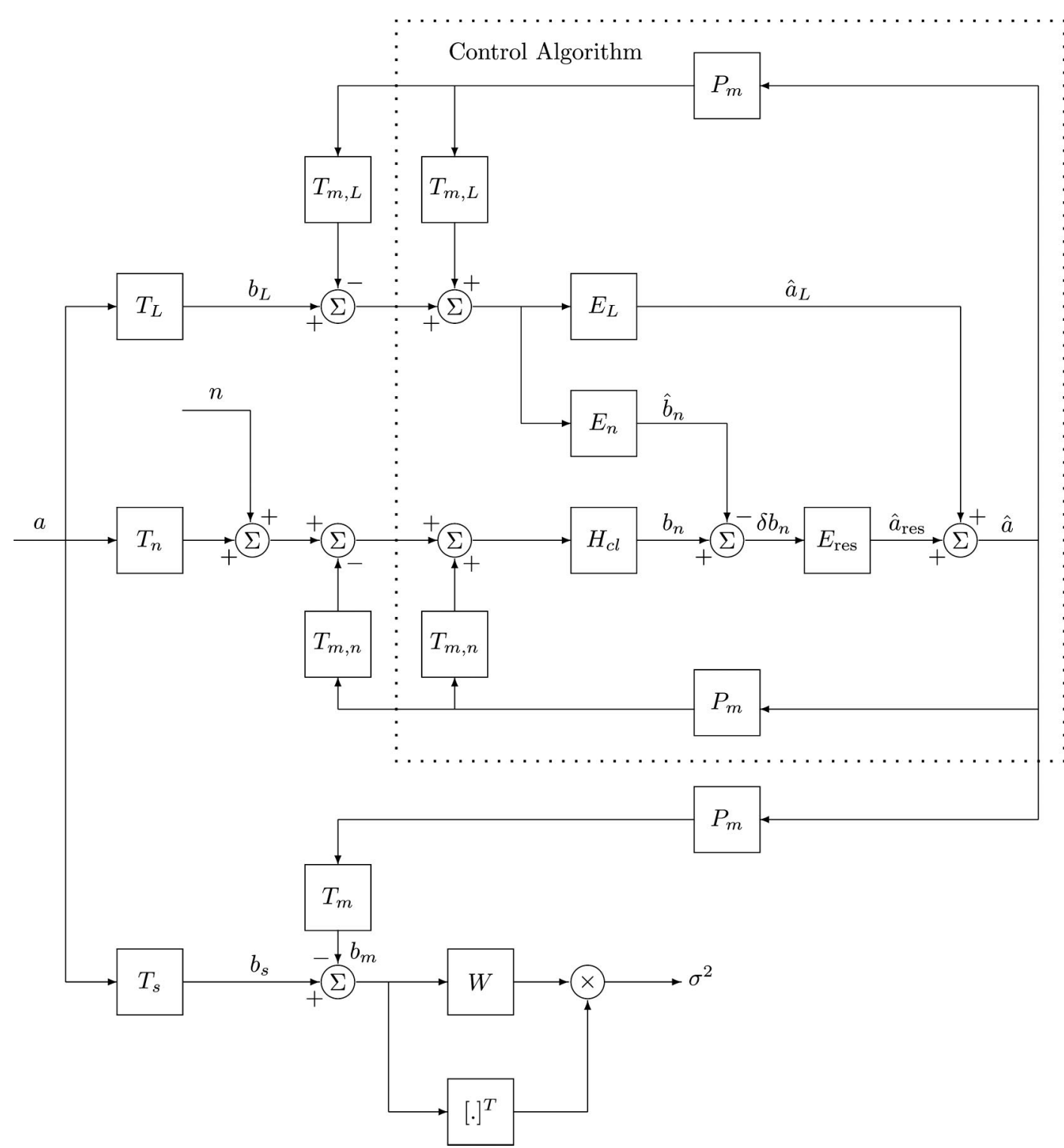

Fig. 1. AO control system model used in this paper. Inside the dotted box is the control algorithm. See the text for the matrix definitions.

uating their performance, are described in the following subsections.

C. Minimum Variance Estimators

The minimum variance estimator, $E_{L}$, of the atmospheric modes, $a$, from the LGS measurements $b_{L}$ is given by ${ }^{11}$

$$
\hat{a}_{L}=\left\langle a b_{L}^{T}\right\rangle\left\langle b_{L} b_{L}^{T}\right\rangle^{\dagger} b_{L}=E_{L} T_{L} a
$$

where

$$
E_{L}=\left\langle a a^{T}\right\rangle T_{L}^{T}\left(T_{L}\left\langle a a^{T}\right\rangle T_{L}^{T}\right)^{\dagger}
$$

and $\dagger$ is the pseudoinverse operator. Similarly, the minimum variance estimator, $E_{n}$, of the NGS mea- surements, $b_{n}$, from the LGS measurements, $b_{L}$, is given by

$$
\hat{b}_{n}=\left\langle b_{n} b_{L}^{T}\right\rangle\left\langle b_{L} b_{L}^{T}\right\rangle^{\dagger} b_{L}=E_{n} T_{L} a,
$$

where

$$
E_{n}=T_{n}\left\langle a^{\prime} a^{T}\right\rangle T_{L}^{T}\left(T_{L}\left\langle a a^{T}\right\rangle T_{L}^{T}\right)^{\dagger} .
$$

Equations (16) and (18) may be combined as

$$
\left(\begin{array}{l}
\hat{a}_{L} \\
\hat{b}_{n}
\end{array}\right)=\left\langle\left(\begin{array}{l}
a \\
b_{n}
\end{array}\right) b_{L}{ }^{T}\right\rangle\left\langle b_{L} b_{L}{ }^{T}\right\rangle^{\dagger} b_{L} .
$$

The mean-squared difference between the atmospheric modes, $a$, and the estimated atmospheric modes, $\hat{a}_{L}$, and the NGS measurements, $b_{n}$, and the estimate of the NGS measurements, $\hat{b}_{n}$ is 


$$
\begin{aligned}
& \left\langle\left(\begin{array}{c}
a_{\mathrm{res}} \\
\delta b_{n}
\end{array}\right)\left(\begin{array}{c}
a_{\mathrm{res}} \\
\delta b_{n}
\end{array}\right)^{T}\right\rangle=\left\langle\left(\begin{array}{c}
a-\hat{a}_{L} \\
b_{n}-\hat{b}_{n}
\end{array}\right)\left(\begin{array}{c}
a-\hat{a}_{L} \\
b_{n}-\hat{b}_{n}
\end{array}\right)^{T}\right\rangle \\
& =\left\langle\left(\begin{array}{l}
a \\
b_{n}
\end{array}\right)\left(\begin{array}{l}
a \\
b_{n}
\end{array}\right)^{T}\right\rangle-\left\langle\left(\begin{array}{l}
a \\
b_{n}
\end{array}\right) b_{L}{ }^{T}\right\rangle\left\langle b_{L} b_{L}{ }^{T}\right\rangle^{\dagger}\left\langle b_{L}\left(\begin{array}{l}
a \\
b_{n}
\end{array}\right)^{T}\right\rangle \\
& =\left[\begin{array}{cc}
\left\langle a a^{T}\right\rangle-\left\langle a b_{L}{ }^{T}\right\rangle\left\langle b_{L} b_{L}{ }^{T}\right\rangle^{\dagger}\left\langle b_{L} a^{T}\right\rangle & \left\langle a b_{n}{ }^{T}\right\rangle-\left\langle a b_{L}{ }^{T}\right\rangle\left\langle b_{L} b_{L}{ }^{T}\right\rangle^{\dagger}\left\langle b_{L} b_{n}{ }^{T}\right\rangle \\
\left\langle b_{n} a^{T}\right\rangle-\left\langle b_{n} b_{L}{ }^{T}\right\rangle\left\langle b_{L} b_{L}{ }^{T}\right\rangle^{\dagger}\left\langle b_{L} a^{T}\right\rangle & \left\langle b_{n} b_{n}{ }^{T}\right\rangle-\left\langle b_{n} b_{L}{ }^{T}\right\rangle\left\langle b_{L} b_{L}{ }^{T}\right\rangle^{\dagger}\left\langle b_{L} b_{n}{ }^{T}\right\rangle
\end{array}\right] .
\end{aligned}
$$

The four terms of this block matrix are the covariance matrices needed to define and evaluate the secondstage minimal variance estimator $E_{\text {res }}$. The computation of the new terms appearing in Eq. (21) are summarized below.

The covariance of the NGS measurements is found from substituting Eq. (5) into $\left\langle b_{n} b_{n}{ }^{T}\right\rangle$ :

$$
\left\langle b_{n} b_{n}{ }^{T}\right\rangle=T_{n}\left\langle a^{\prime} a^{\prime T}\right\rangle T_{n}{ }^{T}+C_{N},
$$

where $C_{N}=\left\langle n^{\prime} n^{\prime T}\right\rangle$ is the covariance of the temporally filtered noise and is discussed in Subsection 3.D. Next, the covariance of the NGS measurements, $b_{n}$, with the atmospheric turbulence, $a$, is similarly given by

$$
\left\langle b_{n} a^{T}\right\rangle=T_{n}\left\langle a^{\prime} a^{T}\right\rangle .
$$

The covariance of the LGS measurements, $b_{L}$, with the NGS measurements, $b_{n}$, is found by substituting Eqs. (4) and (5) into $\left\langle b_{n} b_{L}{ }^{T}\right\rangle$ : $\gamma(i)$ is the strength of layer $i$, and $C$ is a normalized covariance matrix for a single phase screen with unit strength. The covariance of the filtered atmospheric modes with the nonfiltered atmospheric modes, $C_{\phi}{ }^{\prime}$ $=\left\langle a^{\prime} a^{T}\right\rangle$, is a similarly defined block-diagonal matrix where the filtered covariance of the $i$ th layer is

$$
C_{\phi}{ }^{\prime}(i)=\left\langle a(i)^{\prime} a(i)^{T}\right\rangle=\left\langle a(i) a(i)^{\prime T}\right\rangle .
$$

The covariance of the filtered atmospheric modes, $C_{\phi}{ }^{\prime \prime}=\left\langle a^{\prime} a^{\prime T}\right\rangle$, is again a block-diagonal matrix where the filtered covariance of the $i$ th layer is

$$
C_{\phi}^{\prime \prime}(i)=\left\langle a(i)^{\prime} a(i)^{\prime T}\right\rangle .
$$

The calculation of $C_{\phi}{ }^{\prime}(i)$ and $C_{\phi}{ }^{\prime \prime}(i)$ is discussed in Subsection 3.E.

By applying Eqs. (22)-(25), (27), and (28) to Eq. (21) for the covariances of the measurements and Eqs. (17) and (19) for $E_{L}$ and $E_{n}$, we obtain

$$
\left\langle\left(\begin{array}{c}
a-\hat{a}_{L} \\
b_{n}-\hat{b}_{n}
\end{array}\right)\left(\begin{array}{c}
a-\hat{a}_{L} \\
b_{n}-\hat{b}_{n}
\end{array}\right)^{T}\right\rangle=\left[\begin{array}{cc}
C_{\phi}-E_{L} T_{L} C_{\phi} & C_{\phi}{ }^{\prime} T_{n}{ }^{T}-C_{\phi} T_{L}{ }^{T} E_{n}{ }^{T} \\
T_{n} C_{\phi}{ }^{\prime}-E_{n} T_{L} C_{\phi} & T_{n} C_{\phi}{ }^{\prime \prime} T_{n}{ }^{T}-E_{n} T_{L} C_{\phi}{ }^{\prime} T_{n}{ }^{T}+C_{N}
\end{array}\right] \equiv\left[\begin{array}{cc}
A & B^{T} \\
B & D+C_{N}
\end{array}\right] .
$$

$$
\left\langle b_{n} b_{L}^{T}\right\rangle=T_{n}\left\langle a^{\prime} a^{T}\right\rangle T_{L}^{T} .
$$

The covariance matrix of the atmospheric modes, $C_{\phi}=\left\langle a a^{T}\right\rangle$, is a block diagonal matrix with the $i$ th block representing the covariance of the $i$ th layer of the atmosphere:

$$
C_{\phi}=\left[\begin{array}{ccccc}
C_{\phi}(1) & & & & 0 \\
& \ddots & & & \\
& & C_{\phi}(i) & & \\
& & & \ddots & \\
0 & & & & C_{\phi}\left(N_{l}\right)
\end{array}\right],
$$

where there are $i=1, \ldots, N_{l}$ layers of turbulence, and the covariance of the $i$ th layer is given by

$$
C_{\phi}(i)=\left\langle a(i) a(i)^{T}\right\rangle=\gamma(i) C,
$$

The residual error in estimating $a_{\text {res }}=a-\hat{a}_{L}$ from $\delta b_{n}=b_{n}-\hat{b}_{n}$ is given by the minimum variance estimator:

$$
\hat{a}_{\text {res }}=\left\langle a_{\text {res }} \delta b_{n}{ }^{T}\right\rangle\left\langle\delta b_{n} \delta b_{n}{ }^{T}\right\rangle^{-1} \delta b_{n}=E_{\text {res }} \delta b_{n},
$$

where the estimator, $E_{\text {res }}$, is given by substitution of the terms in Eq. (29) in Eq. (30),

$$
E_{\mathrm{res}}=B^{T}\left(D+C_{N}\right)^{-1} .
$$

Finally, the mean-squared difference between the residual atmospheric modes, $a_{\text {res }}$, and the estimate of the residual atmospheric modes, $\hat{a}_{\text {res }}$, is given by

$$
\begin{aligned}
\left\langle\left(a_{\text {res }}-\hat{a}_{\text {res }}\right)\left(a_{\text {res }}-\hat{a}_{\text {res }}\right)^{T}\right\rangle= & A-E_{\text {res }} B-B^{T} E_{\text {res }}{ }^{T} \\
& +E_{\text {res }}\left(D+C_{N}\right) E_{\text {res }}^{T} \\
= & A-B^{T}\left(D+C_{N}\right)^{-1} B .
\end{aligned}
$$


Subtracting Eq. (11) from Eq. (10) shows that

$$
a-\hat{a}=a_{\text {res }}-\hat{a}_{\text {res }},
$$

so that Eq. (32) actually describes the overall estimation error in the two-step estimation algorithm:

$$
\left\langle(a-\hat{a})(a-\hat{a})^{T}\right\rangle=A-B^{T}\left(D+C_{N}\right)^{-1} B .
$$

It is insightful to deconstruct the estimation error calculated in Eq. (35) into individual errors for noise, tilt anisoplanatism, and servo lag. The mean-squared contribution from the NGS measurement noise to the estimate of the atmospheric modes, $\Sigma_{N}$, is given from the noise-covariance-dependent term in Eq. (32):

$$
\Sigma_{N}=\left\langle\left(E_{\text {res }} n^{\prime}\right)\left(E_{\text {res }} n^{\prime}\right)^{T}\right\rangle=\left(E_{\text {res }} C_{N} E_{\text {res }}{ }^{T}\right) .
$$

Next, the mean-squared error due to tilt anisoplanatism, $\Sigma_{\alpha}$, can be found by replacing the filtered atmospheric covariance terms $\left(C_{\phi}{ }^{\prime}\right.$ and $\left.C_{\phi}{ }^{\prime \prime}\right)$ of $B$ and $D$ of Eq. (29) with the unfiltered atmospheric covariance matrix $\left(C_{\phi}\right)$ such that

$$
\begin{aligned}
& B_{a}=T_{n} C_{\phi}-E_{n} T_{L} C_{\phi}, \\
& D_{a}=T_{n} C_{\phi} T_{n}{ }^{T}-E_{n} T_{L} C_{\phi} T_{n}{ }^{T} .
\end{aligned}
$$

This substitution directly assumes that the NGS WFS measurements are noise free and are not temporally filtered. The mean-squared difference between the atmospheric modes and the estimate from these ideal measurements, $\hat{a}_{a}$, is then given by substituting Eqs. (37) and (38) for $B_{a}$ and $D_{a}$ into Eq. (32) and ignoring the noise covariance term:

$$
\begin{aligned}
\Sigma_{a} & =\left\langle\left(a-\hat{a}_{a}\right)\left(a-\hat{a}_{a}\right)^{T}\right\rangle \\
& =A-E_{\mathrm{res}} B_{a}-B_{a}{ }^{T} E_{\mathrm{res}}{ }^{T}+E_{\mathrm{res}} D_{a} E_{\mathrm{res}}{ }^{T} .
\end{aligned}
$$

Finally, the mean-squared error due to servo lag, $\Sigma_{l}$ is then defined as the remaining error, namely,

$$
\begin{aligned}
\Sigma_{l} & =\left\langle(a-\hat{a})(a-\hat{a})^{T}\right\rangle-\Sigma_{N}-\Sigma_{a} \\
& =\delta A-E_{\mathrm{res}} \delta B-\delta B^{T} E_{\mathrm{res}}{ }^{T}+E_{\mathrm{res}} \delta D E_{\mathrm{res}}{ }^{T},
\end{aligned}
$$

where

$$
\begin{aligned}
& \delta A=0, \\
& \delta B=T_{n}\left(C_{\phi}{ }^{\prime}-C_{\phi}\right), \\
& \delta D=T_{n}\left(C_{\phi}{ }^{\prime \prime}-C_{\phi}\right) T_{n}{ }^{T}-E_{n} T_{L}\left(C_{\phi}{ }^{\prime}-C_{\phi}\right) T_{n}{ }^{T},
\end{aligned}
$$

such that $A+\delta A=A, B_{a}+\delta B=B$, and $D_{a}+\delta D$ $=D$.

\section{Residual Phase Variance Formulas}

The estimation error covariance matrix $\langle(a-\hat{a})(a$ $\left.-\hat{a})^{T}\right\rangle$ defines the error in estimating the atmospheric turbulence profile not the wavefront error for the science instrument. In Ref. 11, we derive the meansquared wavefront error $\left(\operatorname{rad}^{2}\right)$ for a MOAO system as

$$
\sigma_{\mathrm{MOAO}}{ }^{2}=\operatorname{Tr}\left[T_{s}^{T} W T_{s}\left\langle(a-\hat{a})(a-\hat{a})^{T}\right\rangle\right],
$$

where $\operatorname{Tr}$ is the trace of the matrix. If we substitute Eq. (35) for $\left\langle(a-\hat{a})(a-\hat{a})^{T}\right\rangle$ in Eq. (44), we obtain

$$
{\sigma_{\mathrm{MOAO}}}^{2}=\operatorname{Tr}\left\{T_{s}^{T} W T_{s}\left[A-B^{T}\left(D+C_{N}\right)^{-1} B\right]\right\} .
$$

For a MCAO system, the residual phase error $\left(\mathrm{rad}^{2}\right)$ is the sum of the error in estimating the wavefront and the error in fitting the estimated modes to the DMs:

$$
\sigma_{\mathrm{MCAO}}^{2}=\sigma_{\text {est }}^{2}+\sigma_{\text {fit }}^{2} .
$$

The fitting and estimation errors are, respectively, ${ }^{11}$

$$
\begin{gathered}
{\sigma_{\text {fit }}}^{2}=\operatorname{Tr}\left[\left(T_{s}-T_{m} P_{m}\right)^{T} W\left(T_{s}-T_{m} P_{m}\right) C_{\phi}\right], \\
{\sigma_{\text {est }}}^{2}=\operatorname{Tr}\left[\left(T_{m} P_{m}\right)^{T} W\left(T_{m} P_{m}\right)\left\langle(a-\hat{a})(a-\hat{a})^{T}\right\rangle\right] .
\end{gathered}
$$

If we substitute Eq. (35) for $\left\langle(a-\hat{a})(a-\hat{a})^{T}\right\rangle$ in Eq. (48), we obtain the estimation error for MCAO:

$$
{\sigma_{\text {est }}}^{2}=\operatorname{Tr}\left\{\left(T_{m} P_{m}\right)^{T} W\left(T_{m} P_{m}\right)\left[A-B^{T}\left(D+C_{N}\right)^{-1} B\right]\right\} .
$$

The wavefront errors due to noise, tilt anisoplanatism, and servo lag, respectively, can similarly be found by substituting Eqs. (36), (39), and (40) into Eq. (44) for MOAO and Eq. (48) for MCAO, respectively.

For both MOAO and MCAO modes, the wavefront error, $\sigma$, in nanometers of the phase is often more convenient. The conversion from $\sigma^{2}\left(\operatorname{rad}^{2}\right)$ to $\sigma(\mathrm{m})$ is simply

$$
\sigma(\mathrm{m})=\frac{\sqrt{\sigma^{2}\left(\mathrm{rad}^{2}\right)}}{2 \pi / \lambda_{e}},
$$

where $\lambda_{e}$ is the $r_{0}$ evaluation wavelength.

\section{Computational Details}

\section{A. Wavefront Reconstruction Error due to Noise for a Low-Order Shack-Hartmann Wavefront Sensor}

In this paper, we compare the noise performance of a quad-cell SH WFS ${ }^{15}$ against a more complex sensor estimating the displacements of subaperture NGS images using a matched-filter ${ }^{16}$ (or noise-weighted least-squares) approach. In this subsection, we calculate the covariance matrix of estimated Zernike coefficients for the SH WFS. We consider the cases of the NGS WFS measuring the first radial order of Zernike polynomials (i.e., tip and tilt) with a single 
subaperture and the NGS WFS measuring the first two orders of Zernike polynomials [i.e., tip, tilt, focus, and the astigmatism modes (TTFA)], which requires a $2 \times 2$ array of subapertures. For the former approach, the number of Zernike modes measured, $N_{z}$, is 2 , and for the latter approach, $N_{z}=5$. The SH WFS actually measures wavefront gradients that must be reconstructed (or scaled) into these low-order wavefront modes. For the ideal gradient WFS model, the wavefront slopes in the $x$ and $y$ directions for the $k$ th NGS, $s_{x}(k)$, and $s_{y}(k)$, are given by

$$
\begin{aligned}
& s_{x}(k)=G_{x} b(k)+\theta_{x}(k), \\
& s_{y}(k)=G_{y} b(k)+\theta_{y}(k),
\end{aligned}
$$

where $G_{x}$ and $G_{y}$ are the interaction matrices of the slopes to the Zernike polynomials, $b(k)$ are the coefficents of the Zernike polynomials for the $k$ th NGS, and $\theta_{x}$ and $\theta_{y}$ are the additive noise on the measurements in the $x$ and $y$ directions. The variance of the one-axis noise for the $k$ th NGS, $\sigma_{\theta}^{2}(k)$, is given by

$$
\sigma_{\theta}^{2}(k)=\left\langle\left|\theta_{x}(k)\right|^{2}\right\rangle=\left\langle\left|\theta_{y}(k)\right|^{2}\right\rangle .
$$

The statistics of the $\theta_{x}(k)$ and $\theta_{y}(k)$ will depend upon the NGS signal level, the WFS read noise, and the particulars of the processing algorithm used to estimate the subaperture TT from the measured intensities.

The sensitivity of the $i$ th subaperture to the $j$ th Zernike polynomial in the $x, G_{x}(i, j)$, and $y$ directions, $G_{y}(i, j)$ is given by

$$
\begin{aligned}
& G_{x}(i, j)= \frac{\frac{\lambda_{e}}{2 \pi} \int_{\mathrm{sa}_{i}} \frac{\partial Z_{j}(x, y)}{\partial x} \mathrm{~d} x \mathrm{~d} y}{\int_{\mathrm{sa}_{i}} 1 \mathrm{~d} x \mathrm{~d} y}, \\
& G_{y}(i, j)=\frac{\frac{\lambda_{e}}{2 \pi} \int_{\mathrm{sa}_{i}} \frac{\partial Z_{j}(x, y)}{\partial y} \mathrm{~d} x \mathrm{~d} y}{\int_{\mathrm{sa}_{i}} 1 \mathrm{~d} x \mathrm{~d} y},
\end{aligned}
$$

where $Z_{j}$ is the $j$ th Zernike polynomial using Noll's ordering approach, ${ }^{13} \lambda_{e}$ is the evaluation wavelength, and the integration domain $\mathrm{sa}_{i}$ is the $i$ th subaperture. By converting to polar coordinates and using Noll's definitions for the derivatives of the Zernikes, ${ }^{13}$ it can be shown that for a single fully illuminated subaperture

$$
\begin{aligned}
G_{x} & =\frac{2 \lambda_{e}}{\pi D}\left[\begin{array}{ll}
1 & 0
\end{array}\right], \\
G_{y} & =\frac{2 \lambda_{e}}{\pi D}\left[\begin{array}{ll}
0 & 1
\end{array}\right],
\end{aligned}
$$

where $D$ is the telescope diameter. Similarly for the $2 \times 2$ fully illuminated subapertures, $G_{x}$ and $G_{y}$ are given by

$$
\begin{gathered}
G_{x}=\frac{2 \lambda_{e}}{\pi D}\left[\begin{array}{ccccc}
1 & 0 & \frac{8}{\sqrt{3} \pi} & \frac{4 \sqrt{6}}{3 \pi} & \frac{4 \sqrt{6}}{3 \pi} \\
1 & 0 & -\frac{8}{\sqrt{3} \pi} & \frac{4 \sqrt{6}}{3 \pi} & -\frac{4 \sqrt{6}}{3 \pi} \\
1 & 0 & -\frac{8}{\sqrt{3} \pi} & -\frac{4 \sqrt{6}}{3 \pi} & -\frac{4 \sqrt{6}}{3 \pi} \\
1 & 0 & \frac{8}{\sqrt{3} \pi} & -\frac{4 \sqrt{6}}{3 \pi} & \frac{4 \sqrt{6}}{3 \pi}
\end{array}\right], \\
G_{y}=\frac{2 \lambda_{e}}{\pi D}\left[\begin{array}{ccccc}
0 & 1 & \frac{8}{\sqrt{3} \pi} & \frac{4 \sqrt{6}}{3 \pi} & -\frac{4 \sqrt{6}}{3 \pi} \\
0 & 1 & \frac{8}{\sqrt{3} \pi} & -\frac{4 \sqrt{6}}{3 \pi} & -\frac{4 \sqrt{6}}{3 \pi} \\
0 & 1 & -\frac{8}{\sqrt{3} \pi} & -\frac{4 \sqrt{6}}{3 \pi} & \frac{4 \sqrt{6}}{3 \pi} \\
0 & 1 & -\frac{8}{\sqrt{3} \pi} & \frac{4 \sqrt{6}}{3 \pi} & \frac{4 \sqrt{6}}{3 \pi}
\end{array}\right] .
\end{gathered}
$$

The noise-weighted least-squares estimate of the Zernike modes, $\hat{b}_{k}$, from the wavefront slopes is

$$
\hat{b}_{k}=\left\{\left[\begin{array}{ll}
G_{x}{ }^{T} & G_{y}{ }^{T}
\end{array}\right] \Theta_{N}{ }^{-1}\left(\begin{array}{l}
G_{x} \\
G_{y}
\end{array}\right)\right\}^{-1}\left[\begin{array}{ll}
G_{x}{ }^{T} & G_{y}{ }^{T}
\end{array}\right] \Theta_{N}{ }^{-1}\left(\begin{array}{l}
s_{x} \\
s_{y}
\end{array}\right),
$$

where $\Theta_{N}$ is the noise covariance matrix in the slope space.

The covariance matrix of the phase estimation error due to the noise of the $k$ th $\operatorname{NGS~} \Sigma_{b}^{2}(k)$ is given by

$$
\Sigma_{b}^{2}(k)=\sigma_{\theta}^{2}(k)\left\{\left[\begin{array}{ll}
G_{x}^{T} & G_{y}^{T}
\end{array}\right]\left(\begin{array}{l}
G_{x} \\
G_{y}
\end{array}\right)\right\}^{-1}
$$

where for $1 \times 1$ subapertures

$$
\left\{\left[\begin{array}{ll}
G_{x}{ }^{T} & G_{y}{ }^{T}
\end{array}\right]\left(\begin{array}{l}
G_{x} \\
G_{y}
\end{array}\right)\right\}^{-1}=\left(\frac{\pi D}{2 \lambda_{e}}\right)^{2}\left[\begin{array}{ll}
1 & 0 \\
0 & 1
\end{array}\right],
$$


and for $2 \times 2$ subapertures

$$
\left\{\left[\begin{array}{ll}
G_{x}{ }^{T} & G_{y}{ }^{T}
\end{array}\right]\left(\begin{array}{l}
G_{x} \\
G_{y}
\end{array}\right)\right\}^{-1}=\left(\frac{\pi D}{4 \lambda_{e}}\right)^{2}\left[\begin{array}{ccccc}
1 & 0 & 0 & 0 & 0 \\
0 & 1 & 0 & 0 & 0 \\
0 & 0 & \frac{3 \pi^{2}}{128} & 0 & 0 \\
0 & 0 & 0 & \frac{3 \pi^{2}}{64} & 0 \\
0 & 0 & 0 & 0 & \frac{3 \pi^{2}}{64}
\end{array}\right] .
$$

The noise covariance matrix, $C_{N}$, is a diagonal matrix and is found by expanding Eq. (91) for multiple NGS:

$C_{N}=\psi\left(f_{s}\right)\left[\begin{array}{ccccc}\Sigma_{b}^{2}(1) & & & & 0 \\ & \ddots & & & \\ & & \Sigma_{b}^{2}(k) & & \\ & & & \ddots & \\ 0 & & & & \Sigma_{b}^{2}\left(N_{\mathrm{NGS}}\right)\end{array}\right]$,

where the matrices $\Sigma_{b}^{2}(k)$ are diagonal for the case where first- or second-radial-order Zernikes are reconstructed from a SH WFS with $1 \times 1$ or $2 \times 2$ subapertures, respectively, and $\psi$ is the noise gain.

\section{B. Tip-Tilt Measurement Noise for a Quadrant Detector}

Here, we describe a model for the noise equivalent angle, $\sigma_{\theta}$, assuming a NGS SH WFS with quad-cell detectors. ${ }^{17}$ The rms one-axis TT measurement error on each subaperture (alternatively, the noise equivalent angle) is given by

$$
\sigma_{\theta}=\frac{\theta_{B}}{\mathrm{SNR}}
$$

where $\theta_{B}$ is the effective spot size of the subaperture NGS image, and SNR is the signal-to-noise ratio of a single subaperture. For a quadrant detector, the SNR is given by

$$
\mathrm{SNR}=\frac{N_{p}}{\sqrt{N_{p}+4 N_{b}+4 \sigma_{e}^{2}}},
$$

where $N_{p}$ is the number of photodetection events per subaperture, $N_{b}$ is the number of background photodetection events per subaperture, and $\sigma_{e}$ is the rms detector read noise per pixel.

In the optical ( $V$ band), we assume that the NGS images are effectively seeing limited, and the effective spot size is given by ${ }^{17}$

$$
\theta_{B}=\frac{\lambda}{4 r_{0} \int_{0}^{\infty} \exp \left(-3.44 \kappa^{5 / 3}\right) \mathrm{d} \kappa},
$$

where $\lambda$ is the imaging wavelength, and $r_{0}$ is the Fried parameter. ${ }^{18}$ The integral $\int_{0}^{\infty} \exp \left(-3.44 \kappa^{5 / 3}\right) \mathrm{d} \kappa$ is computed numerically, with a value of 0.4258 .

In the IR ( $J$ band), we assume that the NGS images contain a diffraction-limited core, and the effective spot size is given by ${ }^{15}$

$$
\theta_{B}=\frac{3 \pi \lambda \sqrt{N_{\mathrm{sa}}}}{16 D}
$$

where $N_{\text {sa }}$ is the total number of subapertures for the NGS WFS.

The number of photons per subaperture is given by ${ }^{17}$

$$
N_{p}=\frac{z 10^{-m / 2.5} \chi t_{s} A_{T}}{N_{\mathrm{sa}}},
$$

where $z$ is the intensity of a 0 magnitude star, $m$ is the magnitude of the NGS, $\chi$ is the end-to-end efficiency of the optics and WFS detectors, $t_{s}$ is the integration time, and $A_{T}$ is the area of the telescope pupil $\left(\pi R^{2}\right)$. The value of $z$, and hence $N_{p}$, is scaled by the Strehl ratio for $J$-band sensing with diffractionlimited pixels, since it is assumed that the size of this quad cell is matched to the diffraction-limited core.

Finally, the number of background photodetection events per pixel in each subaperture is given by ${ }^{17}$

$$
N_{b}=\frac{z_{b} \chi t_{s} A_{T} w^{2}}{N_{\mathrm{sa}}}
$$

where $z_{b}$ is the background intensity per square arc second in the plane of the telescope, and $w$ is the angular subtense of the square pixel.

\section{Subaperture Tip-Tilt Estimation Using a Matched Filter}

In this subsection, we calculate the variance of the unfiltered noise $\Sigma_{b}^{2}$ for the matched-filter (or noiseweighted least-squares) approach. This processing algorithm is applicable if the SH WFS forms actual images of the NGS in each subaperture. The NGS image, $I$, is simply a shifted version of the normalized image $I_{0}$ times the number of photodetection events, $N_{p}$ :

$$
I(r)=N_{p} I_{0}(r+\theta) .
$$

The number of photodetection events, $N_{p}$, is calculated with Eq. (69).

Equivalently, the NGS image is given by

$$
I=N_{p} \mathscr{F}^{-1}\left\{\mathscr{F}_{\{}\left\{I_{0}\right\} \exp (-j 2 \pi \kappa \theta)\right\},
$$

where $\kappa=\left(\kappa_{x}, \kappa_{y}\right)$, and $\mathscr{F}_{F}$ and $\mathscr{F}^{-1}$ are the Fourier and inverse Fourier transforms. The partial derivatives of the NGS images in the $x$ and $y$ directions evaluated at $\theta=0$ are 


$$
\begin{aligned}
& \left.\frac{\partial I}{\partial \theta_{x}}\right|_{\theta=0}=N_{p} \mathscr{F}^{-1}\left\{-j 2 \pi \kappa_{x} \mathscr{F}_{\{}\left\{I_{0}\right\}\right\}, \\
& \left.\frac{\partial I}{\partial \theta_{y}}\right|_{\theta=0}=N_{p} \mathscr{F}^{-1}\left\{-j 2 \pi \kappa_{y} \mathscr{F}_{\{}\left\{I_{0}\right\}\right\} .
\end{aligned}
$$

The interaction matrix for the matched-filter approach, $Q$, is

$$
Q=\left[\begin{array}{ll}
Q_{x} & Q_{y}
\end{array}\right]=\left[\begin{array}{ll}
\frac{\partial I}{\partial \theta_{x}} & \frac{\partial I}{\partial \theta_{y}}
\end{array}\right] .
$$

The matched-filter algorithm for estimating the tilt on the $i$ th NGS image, $\theta_{i}$, from the $i$ th NGS image $I_{i}$ is

$$
\hat{\theta}_{i}=\left(Q^{T} C_{N}{ }^{-1} Q\right)^{-1} Q^{T} C_{N}{ }^{-1}\left(I_{i}-I_{0}\right),
$$

where $C_{N}$, the noise covariance, is the sum of the shot noise contribution $I$, background $N_{b}$, and read noise $\sigma_{e}$ :

$$
C_{N}=I+N_{b}+\sigma_{e}^{2} .
$$

Note that the noise covariance does not include the so-called speckle noise due to the time-varying performance of the AO system and the resulting variability in the NGS WFS point-spread function. The background noise, $N_{b}$, is calculated using Eq. (70). The variance of the unfiltered measurement noise for an on-axis NGS is given by

$$
\Sigma_{b}^{2}(0)=\left(Q^{T} C_{N}{ }^{-1} Q\right)^{-1} .
$$

The $x$ and $y$ coordinates of the noise will have unequal variances if the NGS images are asymmetrical. If the location of the NGS in the FOV is at an angle of $\alpha$ to the $x$ axis, the noise covariance matrix may be computed using

$$
\Sigma_{b}^{2}(\alpha)=T_{\alpha} \Sigma_{b}^{2}(0) T_{\alpha}{ }^{T},
$$

where the rotation matrix, $T_{\alpha}$, is of the standard form:

$$
T_{\theta}=\left[\begin{array}{cc}
\cos \alpha & \sin \alpha \\
-\sin \alpha & \cos \alpha
\end{array}\right] .
$$

D. Natural Guide Star Wavefront Sensor Measurement Noise Filtering

The atmospheric Zernike coefficients measured by the NGS WFS are given by Eq. (5), which is repeated here

$$
b_{n}=T_{n} a^{\prime}+n^{\prime},
$$

where $n^{\prime}$ is the temporally filtered additive noise on the NGS WFS measurements. As mentioned previously, the NGS measurements are temporally filtered by the open-loop transfer function $H_{\mathrm{ol}}(s)$. The noise transfer function, $H_{n}(s)$, is defined by

$$
H_{n}(s)=\frac{H_{\mathrm{cl}}(s)}{H_{\mathrm{WFS}}(s)},
$$

where $H_{\mathrm{WFS}}(s)$ is the sample-and-hold WFS transfer function and is given by ${ }^{19}$

$$
H_{\mathrm{WFS}}(s)=\frac{f_{s}}{s}\left[1-\exp \left(\frac{-s}{f_{s}}\right)\right] .
$$

The filtered noise signal with respect to time, $n^{\prime}(t)$, is the convolution of the noise impulse response, $h_{n}(t)$, with the unfiltered noise signal, $n(t)$ :

$$
n^{\prime}(t)=n(t) \circ h_{n}(t) .
$$

Using the convolution theorem in Eq. (84) yields,

$$
N^{\prime}(f)=N(f) H_{n}(f),
$$

where $f$ is the frequency domain coordinate. We take the inverse Fourier transform of Eq. (85) to get the filtered noise signal as a function of the unfiltered noise spectrum:

$$
n^{\prime}(t)=\int_{-\infty}^{\infty} N(f) \exp (j 2 \pi f t) H_{n}(f) \mathrm{d} f,
$$

and $j=\sqrt{-1}$. The ensemble mean-squared average of the filtered noise is given by multiplying Eq. (86) by its complex conjugate and taking the ensemble:

$$
\left\langle\left|n^{\prime}(t)\right|^{2}\right\rangle=\int_{-\infty}^{\infty}\left\langle|N(f)|^{2}\right\rangle\left|H_{n}(f)\right|^{2} \mathrm{~d} f .
$$

The noise spectrum $N(f)$ is only nonzero between $-f_{s} / 2$ and $f_{s} / 2$, where $f_{s}$ is the sampling frequency of the NGS WFS. If we also assume that $\left\langle|N(f)|^{2}\right\rangle$ is constant with respect to $f$ within this passband, then

$$
\left\langle\left|n^{\prime}(t)\right|^{2}\right\rangle=\left\langle|N(f)|^{2}\right\rangle \int_{-f_{s} / 2}^{f_{s} / 2}\left|H_{n}(f)\right|^{2} \mathrm{~d} f .
$$

The WFS sample time, $t_{s}$, is defined by

$$
t_{s}=\frac{1}{f_{s}} \text {. }
$$

The unfiltered noise spectrum is given by

$$
\left\langle|N(f)|^{2}\right\rangle=t_{s} \Sigma_{b}^{2}=\frac{\Sigma_{b}^{2}}{f_{s}}
$$

where $\Sigma_{b}^{2}$ is the variance of the unfiltered measurement noise on a single measurement as derived in 
Subsection 3.A for a quad cell and Subsection 3.C for the matched filter. Substituting Eq. (90) into Eq. (88) gives

$$
\left\langle\left|n^{\prime}(t)\right|^{2}\right\rangle=\frac{\Sigma_{b}^{2}}{f_{s}} \int_{-f_{s} / 2}^{f_{s} / 2}\left|H_{n}(f)\right|^{2} \mathrm{~d} f=\Sigma_{b}^{2} \psi\left(f_{s}\right),
$$

where the noise gain, $\psi\left(f_{s}\right)$, is given by

$$
\psi\left(f_{s}\right)=\frac{1}{f_{s}} \int_{-f_{s} / 2}^{f_{s} / 2}\left|H_{n}(f)\right|^{2} \mathrm{~d} f .
$$

The noise gain due to the temporal filtering is thus a function of the sampling frequency of the NGS WFS.

\section{E. Covariance of Temporally Filtered Turbulence}

Noll defines the covariance of the atmosphere in terms of the Zernikes, $a$, as ${ }^{13}$

$$
\begin{aligned}
\left\langle a_{n m} a_{n^{*} m^{*}}{ }^{T}\right\rangle= & (0.046 / \pi)\left(R / r_{0}\right)^{5 / 3}\left[(n+1)\left(n^{*}+1\right)\right]^{1 / 2} \\
& \times(-1)^{\left(n+n^{*}-2 n\right) / 2} \delta_{m m^{*}} I_{n n^{*}},
\end{aligned}
$$

where in Ref. 11, we show that for a finite-outer scale, $L_{0}$, the term $I_{n n *}$ is given by

$$
I_{n n^{*}}=\int_{0}^{\infty} \kappa^{-1} \frac{J_{n+1}(2 \pi \kappa) J_{n^{*}+1}(2 \pi \kappa)}{\left[\kappa^{2}+\left(\frac{R}{L_{0}}\right)^{2}\right]^{11 / 6}} \mathrm{~d} \kappa,
$$

where $J_{n}$ is an $n$ th-order Bessel function of the first kind, $n$ and $m$ are the radial and azimuthal orders of the Zernikes, respectively, $R$ is the telescope radius, and $\kappa$ is the coordinate in the Fourier space. Equation (94) was derived by including the von Karman spectrum in Noll's original definition of $I_{n n}$. Similar formulas are derived here for the temporally filtered covariances $\left\langle a^{\prime} a^{T}\right\rangle$ and $\left\langle a^{\prime} a^{\prime T}\right\rangle$ assuming one particular wind-speed model again using power spectral techniques.

The closed-loop transfer function, $H_{\mathrm{cl}}(s)$, of the temporal filter is given by

$$
H_{\mathrm{cl}}(s)=\frac{H_{\mathrm{ol}}(s)}{1+H_{\mathrm{ol}}(s)}
$$

In Ref. 20, it is shown that for a wind profile with a known wind speed and a random wind direction uniformly distributed between 0 and $2 \pi$, the cross statistics between the spectra of the filtered and unfiltered phase profiles is given by

$$
\left\langle\hat{\phi}^{\prime}(i ; \kappa) \hat{\phi}^{*}(i ; \kappa)\right\rangle=\left\langle|\hat{\phi}(i ; \kappa)|^{2}\right\rangle h^{\prime}(\kappa ; i),
$$

where $\left\langle|\hat{\phi}(i ; \kappa)|^{2}\right\rangle$ is the power spectrum of the $i$ th layer of the unfiltered atmosphere, and

$$
h^{\prime}(\kappa ; i)=\int_{0}^{2 \pi} H_{\mathrm{cl}[}[j v(i) \kappa \cos (\theta)] \mathrm{d} \theta,
$$

where we have made the substitution $s=j v(i) \kappa \cos \theta$, and $v(i)$ is the wind speed at the $i$ th atmospheric layer. Similarly, the power spectrum of the temporally filtered atmosphere, $\left\langle\left|\hat{\phi}^{\prime \prime}(i ; \kappa)\right|^{2}\right\rangle$, is

$$
\left\langle\left|\hat{\phi}^{\prime \prime}(i ; \kappa)\right|^{2}\right\rangle=\left\langle|\hat{\phi}(i ; \kappa)|^{2}\right\rangle h^{\prime \prime}(\kappa ; i),
$$

where

$$
h^{\prime \prime}(\kappa ; i)=\int_{0}^{2 \pi}\left|H_{\mathrm{c}[}[j v(i) \kappa \cos (\theta)]\right|^{2} \mathrm{~d} \theta .
$$

Note that the integrals in Eqs. (97) and (99) arise from the particular form of the wind model assumed here, i.e., a constant wind speed and a random wind direction.

The cross covariance between the filtered and unfiltered Zernike modes $\left\langle a_{n m^{\prime}}{ }^{\prime}(i) a_{n^{*} m^{*}}(i)^{T}\right\rangle$ is now found by using Eq. (96) in a derivation otherwise identical to Noll's development of Eq. (93),

$$
\begin{aligned}
\left\langle a_{n m^{\prime}}(i) a_{n^{*} m^{*}}(i)^{T}\right\rangle= & (0.046 / \pi)\left(R / r_{0}\right)^{5 / 3} \\
& \times\left[(n+1)\left(n^{*}+1\right)\right]^{1 / 2} \\
& \times(-1)^{\left(n+n^{*}-2 n\right) / 2} \delta_{m m^{*}} I_{n n^{*^{\prime}}}(i),
\end{aligned}
$$

where the value of $I_{n n^{*}}(i)$ for the $i$ th layer is given by

$$
\begin{aligned}
I_{n n^{*}}(i)= & \int_{0}^{\pi / 2} \frac{\sec ^{2}(\beta)}{\tan (\beta)} \frac{J_{n+1}[\tan (\beta)] J_{n^{*}+1}[\tan (\beta)]}{\left[\left(\frac{\tan (\beta)}{2 \pi}\right)^{2}+\left(\frac{R}{L_{0}}\right)^{2}\right]^{11 / 6}} \\
& \times h^{\prime}\left[\frac{\tan (\beta) v(i)}{2 \pi R} ; i\right] \mathrm{d} \beta,
\end{aligned}
$$

and we have made the substitution $\tan (\beta)=2 \pi \kappa$ for $\kappa$ in Eqs. (94) and (97). Similarly, the filtered mode covariance is found by using Eq. (98) in Eq. (93):

$$
\begin{aligned}
\left\langle a_{n m}{ }^{\prime}(i) a_{n^{*} m^{*}}(i)^{T}\right\rangle= & (0.046 / \pi)\left(R / r_{0}\right)^{5 / 3} \\
& \times\left[(n+1)\left(n^{*}+1\right)\right]^{1 / 2} \\
& \times(-1)^{\left(n+n^{*}-2 n\right) / 2} \delta_{m m^{*}} I_{n n^{*}}{ }^{\prime \prime}(i),
\end{aligned}
$$

where

$$
\begin{aligned}
I_{n n^{* \prime}}(i)= & \int_{0}^{\pi / 2} \frac{\sec ^{2}(\beta)}{\tan (\beta)} \frac{J_{n+1}[\tan (\beta)] J_{n^{*}+1}[\tan (\beta)]}{\left[\left(\frac{\tan (\beta)}{2 \pi}\right)^{2}+\left(\frac{R}{L_{0}}\right)^{2}\right]^{11 / 6}} \\
& \times h^{\prime \prime}\left[\frac{\tan (\beta) v(i)}{2 \pi R} ; i\right] \mathrm{d} \beta .
\end{aligned}
$$


In this paper, the cross covariance and filtered covariance of the atmosphere, $\left\langle a^{\prime} a^{T}\right\rangle$ and $\left\langle a^{\prime} a^{\prime T}\right\rangle$, are found by integrating Eqs. (101) and (103) numerically, and substituting $I_{n n^{*}}(i)$ and $I_{n n^{* \prime \prime}(i)}$ into Eqs. (100) and (102), respectively. These integrals are numerically difficult when the filter $H_{\mathrm{cl}}$ is optimized to provide good or very good correction.

\section{F. Star Generation}

In the $V$ band, the NGSs are generated using the Bahcall-Soneira star model. ${ }^{21}$ This produces stars up to a magnitude of 30 . In the $J$ band, the NGSs are generated using the Spagna model, ${ }^{22}$ which produces stars with magnitudes up to 22 at the north galactic pole (NGP) and 19 for other galactic latitudes. There are more stars at each magnitude in the $J$ band than in the $V$ band with these models. For example, for a 2 arc min diameter patrol field at the NGP, the median expected magnitude in $J$ is 16.5 and in $V$ is 18.3.

\section{G. Natural Guide Star Sharpening}

The NGSs are expected to be partially corrected (sharpened) by the AO system in the $J$ band. The Strehl of each NGS is a function of its position in the field. Strehl ratios were generated in the Liner AO Simulator (LAOS) ${ }^{14}$ at five points along the $x$ axis and at four points along a line at $30^{\circ}$ to the $x$ axis, although as shown in Fig. 2, there is little angular dependence on the Strehl, and the angular dependence is ignored. This calculation of the Strehl does include the higher-order errors from the LGS such as spot elongation. A cubic fit was made to these nine points as shown in Fig. 2. The Strehl on axis in the $V$ band was calculated using LAOS to be 0.02 , which is significantly less than in the $J$ band justifying the assumption of seeing-limited images in the $V$ band.

\section{$\mathrm{H}$. Wind Shake}

Wind at ground-based telescopes generates TT wavefront errors that are nonnegligible. In this paper, the wind shake is modeled as being statistically independent from atmospheric turbulence, so the total TT error is the sum of the contributions of the two effects.

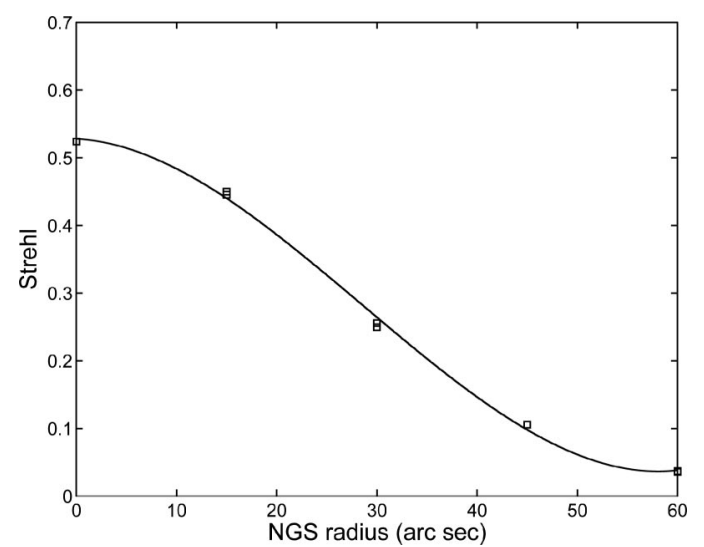

Fig. 2. Strehl ratio in the $J$ band as a function of the position of the NGS in the field. The squares represent the Strehls generated using LAOS, and the curve is a best-fit cubic approximation.

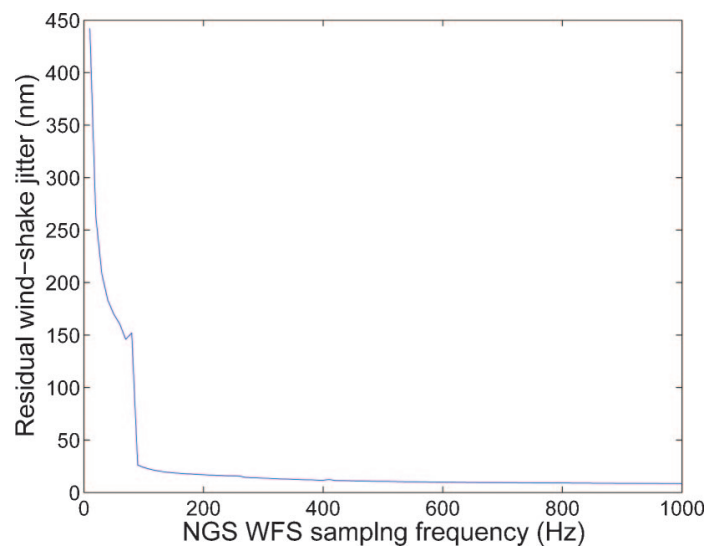

Fig. 3. (Color online) Residual telescope wind-shake tilt jitter after the correction as a function of the sampling frequency of the NGS WFS.

Initial simulations showed that the wind-shake term was the dominant term in the TT error budget for NFIRAOS and that the tip-tilt stage (TTS) alone was insufficient to correct for the TT induced from the wind shake. Instead, we use both the TTS and the DM to correct for the wind shake (woofer-tweeter control). Additionally, we control the TTS with a proportional integral controller such that there are three control paths: the DM, the TTS with a single integrator, and the TTS with two integrators. Each path has a separate gain, which is dependent on the sampling frequency. The woofer-tweeter control of the DM and TTS for NFIRAOS is described in Ref. 19.

The level of uncorrected wind shake assumed for this study is 25 mas, which was calculated from an integrated modeling run of the telescope for a median wind profile. ${ }^{19,23}$ The TTS is assumed to have a 20 $\mathrm{Hz}$ mechanical bandwidth. The residual telescope wind-shake TT jitter after correction by the TTS and DM, $\sigma_{\text {res }}^{2}$, for this model is shown in Fig. 3. The discontinuous nature of this curve is due to the constraints chosen to optimize the gains of the controller (see Ref. 19).

\section{Sodium-Layer Range Estimation Error}

Experimental results have shown that the mean altitude of the sodium layer can vary by several meters per second. ${ }^{10}$ This temporal variation of the sodium layer results in a focus error, which cannot be determined from the sodium LGS WFS, because it cannot be disentangled from the atmospheric focus aberration. Therefore one of the NGSs is required to measure the focus term, and the sampling rate of the TTFA NGS WFS determines the focus error from the sodium-layer altitude variations. So, although the purpose of this paper is to estimate TT errors and not higher-order errors such as this focus error, we also include this error when we optimize the sampling frequency of the NGS WFS.

The calculation of the error in tracking the mean altitude of the sodium layer is presented in Ref. 24 . This focus error calculation is made by extrapolating a power spectrum of height variations from lidar data 


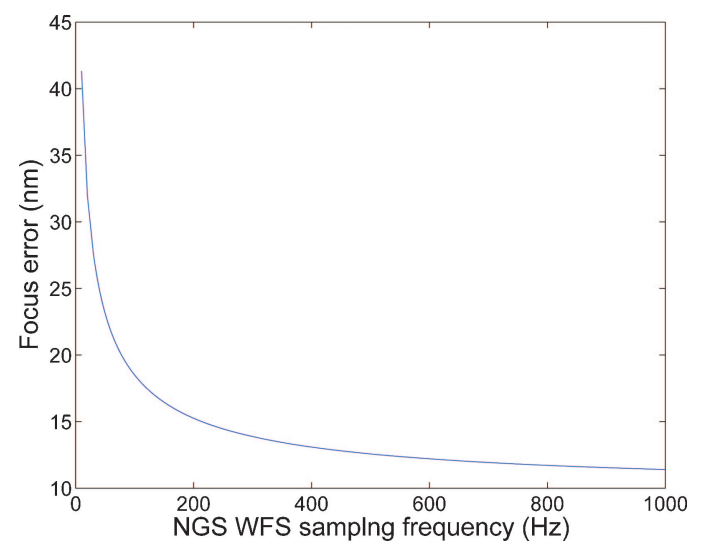

Fig. 4. (Color online) Estimated focus wavefront error due to variations in the mean altitude of the sodium layer as a function of the sampling frequency of the NGS WFS.

and applying the rejection transfer function for using electronic offsets, which will correct for the focus of the LGS WFSs in real time. The residual wavefront focus error, $\sigma_{\mathrm{Na}}$, is plotted versus the NGS WFS sampling frequency in Fig. 4 for the median observed sodium altitude variations.

\section{J. Sampling Frequency Optimization}

The overall wavefront error, $\sigma^{2}\left(\operatorname{rad}^{2}\right)$, is the sum of the atmospheric error $\sigma_{\text {atm }}{ }^{2}$, which is computed with either Eq. (45) for the MOAO mode or Eq. (46) for the MCAO mode; the residual telescope wind-shake jitter, ${\sigma_{\text {res }}}^{2}$; and the sodium layer tracking error, $\sigma_{\mathrm{Na}}{ }^{2}$;

$$
\sigma^{2}\left(f_{s}\right)=\sigma_{\mathrm{atm}}^{2}\left(f_{s}\right)+\sigma_{\mathrm{res}}^{2}\left(f_{s}\right)+\sigma_{\mathrm{Na}}^{2}\left(f_{s}\right)
$$

All the error terms in Eq. (104) are functions of the sampling rate $f_{s}$. We therefore find the optimum sampling frequency, $f_{s}^{*}$, for each NGS constellation with

$f_{s}^{*}=\arg \min _{f_{s}}\left\{\sigma_{\mathrm{atm}}{ }^{2}\left(f_{s}\right)+\sigma_{\mathrm{res}}^{2}\left(f_{s}\right)+\sigma_{\mathrm{Na}}{ }^{2}\left(f_{s}\right)\right\}$.

\section{Narrow-Field Infrared Adaptive Optics System Simulations}

In this section, the sky coverage simulations for the AO system NFIRAOS at the NGP (latitude $=0^{\circ}$, longitude $=90^{\circ}$ ), which represents the worst case for sky coverage, are presented. The turbulence and wind velocity profile used in these simulations are tabulated in Table 1. This profile is generated from measurements obtained at Cerro Pachon. ${ }^{25}$ The other atmospheric and telescope parameters are tabulated in Table 2.

The wavefront errors are evaluated on axis for a single conjugate AO system using Eq. (104). NFIRAOS, in fact, has a 10 arc sec square field in the baseline design and a 30 arc sec square field in the upgrade path, although overall TT performance for these fields is typically within $4 \%$ of the on-axis case.

We investigate both optical ( $V$ band) and IR ( $J$ band) sensing for NFIRAOS. We assume the pixels in
Table 1. Six-Layer Turbulence Profile Typical of Cerro Pachon (Ref. 25) $^{a}$

\begin{tabular}{crcc}
\hline $\begin{array}{c}\text { Layer } \\
i\end{array}$ & $\begin{array}{c}h(i) \\
(\mathrm{m})\end{array}$ & $\gamma(i)$ & $\begin{array}{c}v(i) \\
\left(\mathrm{ms}^{-1}\right)\end{array}$ \\
\hline 1 & 0 & 0.6523 & 5 \\
2 & 2577 & 0.1723 & 13 \\
3 & 5155 & 0.0551 & 20 \\
4 & 7732 & 0.0248 & 30 \\
5 & 12,887 & 0.0736 & 20 \\
6 & 15,464 & 0.0219 & 10 \\
\hline
\end{tabular}

${ }^{a}$ The profile shows the height $h(i)$ of each layer, the relative turbulence strength, $\gamma(i)$, and wind speed, $v(i)$.

the $J$ band are twice diffraction limited (i.e., $w$ in the $J$ band is $\lambda / D$ rads $=0.0086$ arc sec) and seeing limited in the $V$ band ( $w=0.5$ arc sec). The levels of the read noise, $\sigma_{e}$, considered are $0,5,10$, and 15 electrons per pixel per readout. Although the first six Zernike orders are considered in the problem, only the errors arising in the TT terms are evaluated.

Using the $J$ band for the NGS WFS passband yields a conservative bound on the expected sky coverage of an IR TT WFS, since using a wider $J+H$ passband improves TT WFS performance significantly on dim stars. We have not included these results in this paper, since the modeling becomes more complex when AO system performance and the NGS irradiance vary significantly across the wider WFS passband.

The sampling rate of the NGS WFS, $f_{s}$, is optimized for each NGS constellation. The allowable range of

Table 2. System and Atmospheric Parameters

\begin{tabular}{|c|c|c|}
\hline Parameter & Symbol & Value \\
\hline Telescope diameter & $D$ & $30 \mathrm{~m}$ \\
\hline Outer scale & $L_{0}$ & $30 \mathrm{~m}$ \\
\hline Pixel subtense ( $V$ band) & $w$ & 0.5 arc sec \\
\hline Pixel subtense ( $J$ band) & $w$ & $\lambda / D$ rads \\
\hline Fried's parameter & $r_{0}$ & $0.15 \mathrm{~m}$ \\
\hline$r_{0}$ evaluation wavelength & $\lambda_{e}$ & $0.5 \mu \mathrm{m}$ \\
\hline Height of sodium LGS & $H$ & $90 \mathrm{~km}$ \\
\hline Height of Rayleigh LGS & $H_{R}$ & $20 \mathrm{~km}$ \\
\hline DM conjugate altitudes & $h_{m}$ & $0,12 \mathrm{~km}$ \\
\hline $\mathrm{AO}$ order of correction & - & $60 \times 60$ \\
\hline Zernike radial order & $N$ & 6 \\
\hline $\begin{array}{l}\text { End-to-end efficiency of } \\
\text { optics }^{a}\end{array}$ & $x$ & 0.4 \\
\hline $\begin{array}{l}\text { Background intensity }{ }^{b} \\
\text { ( } V \text { band })\end{array}$ & $z_{b}$ & 37.6 photons $\mathrm{m}^{-2}$ arc $\mathrm{sec}^{-2} \mathrm{~s}^{-1}$ \\
\hline $\begin{array}{l}\text { Background intensity }{ }^{b} \\
\quad(J \text { band })\end{array}$ & $z_{b}$ & 1385 photons $\mathrm{m}^{-2} \operatorname{arc~sec}{ }^{-2} \mathrm{~s}^{-1}$ \\
\hline $\begin{array}{l}\text { Intensity of } m=0 \text { star }^{b} \\
\quad(V \text { band })\end{array}$ & $z$ & $9.71 \times 10^{9}$ photons $\mathrm{m}^{-2} \mathrm{~s}^{-1}$ \\
\hline $\begin{array}{l}\text { Intensity of } m=0 \text { star }^{b} \\
\quad(J \text { band })\end{array}$ & $z$ & $5.52 \times 10^{9}$ photons $\mathrm{m}^{-2} \mathrm{~s}^{-1}$ \\
\hline $\begin{array}{l}\text { Imaging wavelength } \\
\text { ( } V \text { band })\end{array}$ & $\lambda$ & $0.5 \mu \mathrm{m}$ \\
\hline $\begin{array}{l}\text { Imaging wavelength } \\
(J \text { band })\end{array}$ & $\lambda$ & $1.25 \mu \mathrm{m}$ \\
\hline
\end{tabular}

${ }^{a}$ Reference 17.

${ }^{b}$ Reference 26. 


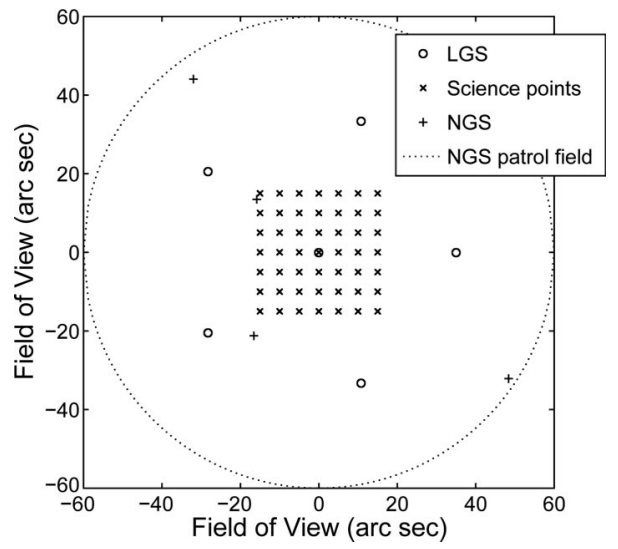

Fig. 5. FOV for the NFIRAOS showing the sodium LGS ( $\bigcirc)$, the 2 arc min diameter patrol field for the NGS (dotted circle), a random constellation of four NGSs (+), and the science evaluation points $(\times)$ for the 30 arc sec square science field.

sampling frequencies is $10-1000 \mathrm{~Hz}$. A single simulation of one NGS constellation takes of the order of $2 \mathrm{~s}$. The majority of this time is spent in optimizing the sampling frequency. The wavefront error, $\sigma^{2}$, for each element in the simulation space is computed for 500 NGS constellations. By reseeding the random number generator, every option in the simulation space is simulated over the same set of 500 NGS constellations.

The baseline NFIRAOS LGS asterism is shown in Fig. 5 and consists of six LGSs: one LGS is on axis, and the remaining five are equally spaced on a ring of a diameter of 70 arc sec. The Rayleigh LGS, when used, is on axis and at a range of $20 \mathrm{~km}$. The science field consists of 49 points arranged in a square grid of a linear dimension of 10 arc sec in the baseline design and 30 arc sec in the upgrade path.

For a given field, a single call of the BahcallSoneira or Spagna models can generate more NGSs than there are NGS WFSs in the NFIRAOS design options. If there are more NGSs in the field than NGS WFSs, the wavefront error for every combination of NGSs is evaluated, and the NGS combination that produces the smallest wavefront error is chosen.

The median TT error versus the patrol band diameter is shown in Fig. 6. When the patrol diameter is less than or equal to 40 arc sec, the median case corresponds to zero NGSs in the field and the openloop TT error of $718 \mathrm{~nm}$. The lowest wavefront error is effectively obtained with a 2 arc min diameter patrol field; stars further away from the science field effectively have too little partial correction and suffer too much anisoplanatism to be useful. We use this diameter for the remainder of the simulations.

In this section, the TT errors are reported in nanometer rms. It is possible to convert the reported errors from nanometers to tilt jitter in milliarc seconds by

$\sigma(\operatorname{mas})=\frac{4 \times 1000 \times 180 \times 60 \times 60}{D \pi} \sigma(m)$.

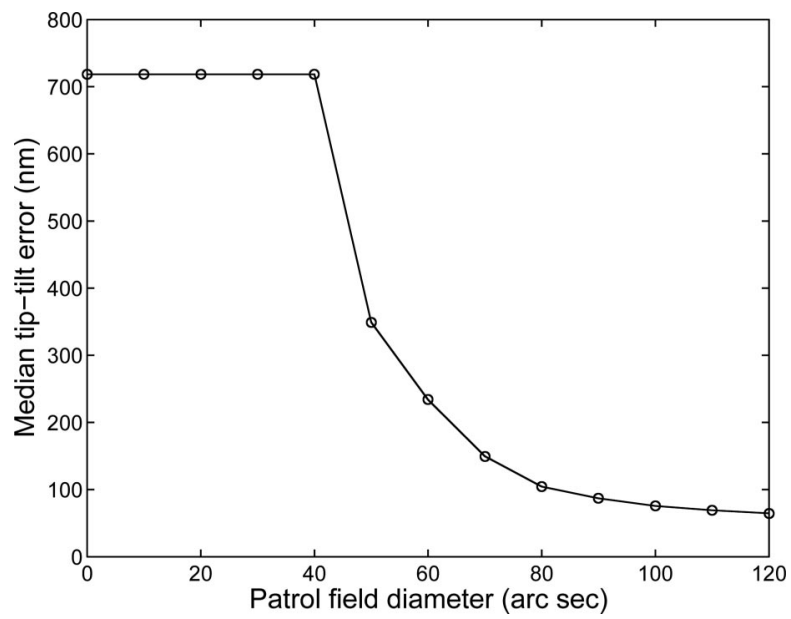

Fig. 6. Median TT error (nm) over 500 NGS constellations versus the patrol field diameter ( $\operatorname{arcsec}$ ) for two TT NGSs and one TTFA NGS in the $J$ band.

For a $30 \mathrm{~m}$ diameter telescope, 1 mas of TT jitter corresponds to $36.4 \mathrm{~nm}$ of TT error.

The median TT errors for the three WFS options in the $J$ and $V$ bands are displayed in Table 3 for $0,5,10$, and 15 electrons of read noise per pixel. The first thing we note from Table 3 is that the results using seeing-limited stars in the $V$ band are, in all cases, significantly worse than using partially compensated stars in the $J$ band in terms of $\sigma(\mathrm{nm})$ and, in fact, fail to reach the current requirements for the NFIRAOS sky coverage. We therefore eliminate using the $V$ band stars and sensors and concentrate on the $J$ band for the remainder of this paper.

There is a clear hierarchy in the performance of the three WFS architectures with the auxiliary Rayleigh LGS the best at all read noise levels, followed by the TTFA and the two TT NGS WFS option, and last, the single TTFA NGS WFS. This introduces a cost versus performance trade-off in the system design with the Rayleigh LGS being nontrivially more complex than the other two options.

The cumulative density function $(\mathrm{CDF}), \operatorname{Pr}(\sigma<\epsilon)$ versus $\epsilon(\mathrm{nm})$, is shown for the three WFS options in the $J$ band in Fig. 7, with ten electrons of read noise, which is the baseline value. We see from Fig. 7 that the auxiliary Rayleigh LGS option always provides a lower TT error than the one TTFA + two TT NGS

Table 3. Median TT Errors (nm) for the NFIRAOS for the Three NGS WFS Options with SH Sensors Using Quad-Cell Detectors

\begin{tabular}{ccrrrr}
\hline & & \multicolumn{4}{c}{$\begin{array}{c}\text { TT Error } \\
(\mathrm{nm})\end{array}$} \\
\cline { 3 - 6 } Band & WFS Option & $\sigma_{e}=0$ & $\sigma_{e}=5$ & $\sigma_{e}=10$ & $\sigma_{e}=15$ \\
\hline \multirow{2}{*}{$V$ band } & One TTFA + two TTs & 371 & 374 & 377 & 386 \\
& One TTFA & 395 & 398 & 409 & 426 \\
& One TT + Rayleigh & 387 & 388 & 391 & 396 \\
$J$ band & One TTFA + two TTs & 43 & 54 & 65 & 76 \\
& One TTFA & 67 & 82 & 103 & 128 \\
& One TT + Rayleigh & 37 & 39 & 42 & 45 \\
\hline
\end{tabular}




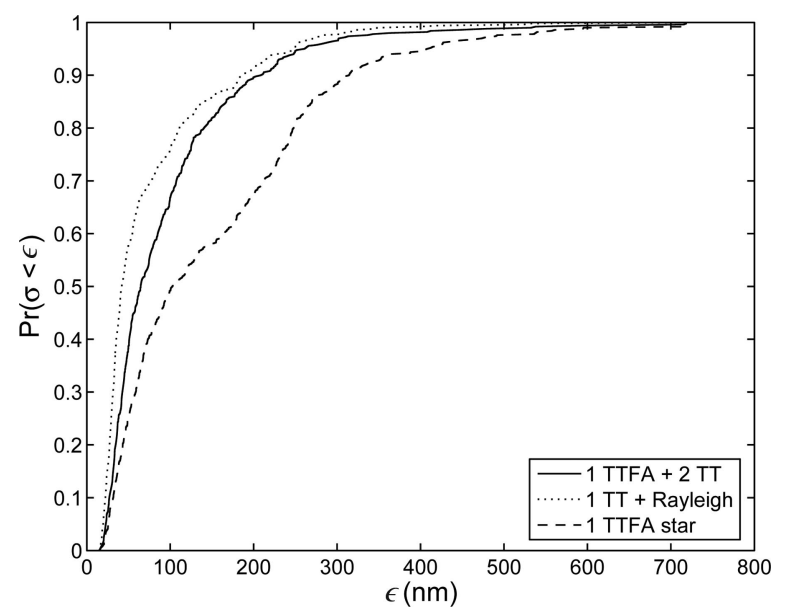

Fig. 7. $\mathrm{CDF}[\operatorname{Pr}(\sigma<\varepsilon)$ versus $\varepsilon(\mathrm{nm})]$ from 500 different NGS constellations in the $J$ band with ten electrons of read noise for the NFIRAOS for one TTFA NGS WFS and two TT NGS WFSs (solid curve), a single TTFA NGS WFS (dashed curve), and a single TT NGS WFS used in conjunction with a Rayleigh LGS (dotted curve).

WFSs option, which always produces a lower TT error than one TTFA NGS WFS.

In Table 4, the median TT error is broken down into wind shake, servo lag, tilt anisoplanatism, and noise on the NGS WFS measurements for the three WFS options with ten electrons of read noise per pixel. Here, the median TT error is the error term averaged over the 20 middle NGS constellations sorted on the total TT error. The median sampling rate is $90 \mathrm{~Hz}$ for the two TT NGS WFSs + one TTFA NGS WFS option, $90 \mathrm{~Hz}$ for the one TTFA NGS WFS, and $140 \mathrm{~Hz}$ for the auxiliary Rayleigh option. The NGS WFS noise is currently the largest term in the error budget for all options, followed by tilt anisoplanatism, wind shake, and servo lag. For the two TT NGS WFSs + one TTFA NGS WFS and one TTFA NGS WFS options, the median sodium tracking error is $19 \mathrm{~nm}$ and $17 \mathrm{~nm}$ for the auxiliary Rayleigh LGS option. For the single TTFA NGS WFS, the median magnitude of the TTFA star is 16.3 , at a median offset of 43 arc sec off axis. For the auxiliary Rayleigh LGS, the median magnitude is 16.1 at a median offset of 44 arc sec off axis. For the two TT NGS WFSs + one

Table 4. Median TT Errors (nm) for the NFIRAOS Broken Down into Wind Shake, Noise, Tilt Anisoplanatism, and Servo Lag for the Three, WFS Options in the $J$ Band $^{a}$

\begin{tabular}{lccc}
\hline & \multicolumn{3}{c}{$\begin{array}{c}\text { Median TT Error } \\
\text { (nm) }\end{array}$} \\
\cline { 2 - 4 } \multicolumn{1}{c}{ Error Source } & Option 1 & Option 2 & Option 3 \\
\hline Wind shake & 26 & 26 & 21 \\
Servo lag & 15 & 15 & 14 \\
Tilt anisoplanatism & 37 & 54 & 21 \\
NGS WFS noise & 44 & 84 & 26 \\
Total & 65 & 104 & 42 \\
\hline
\end{tabular}

${ }^{a}$ Option 1 is one TTFA NGS WFS + two TT NGS WFSs, option 2 is one TTFA NGS WFS, and option 3 is one TT NGS WFS + one Rayleigh LGS. There are ten electrons of read noise per pixel.
Table 5. Median TT Errors (nm) for the NFIRAOS with Three TT NGS WFSs for the Quad-Cell and Matched-Filter Approaches

\begin{tabular}{lccccc}
\hline & \multirow{2}{*}{$\begin{array}{c}\text { Pixel } \\
\text { Detection }\end{array}$} & \multicolumn{5}{c}{$\begin{array}{c}\text { Median TT Error } \\
\text { Subtense } \\
\text { Method }\end{array}$} & (rads) & \multicolumn{5}{c}{} \\
\cline { 3 - 6 } & $\sigma_{e}=0$ & $\sigma_{e}=5$ & $\sigma_{e}=10$ & $\sigma_{e}=15$ \\
\hline Quad cell & $\lambda / D$ & 49 & 65 & 80 & 93 \\
Matched filter & $\lambda / 2 D$ & 31 & 53 & 70 & 79 \\
Matched filter & $\lambda / D$ & 32 & 46 & 56 & 66 \\
Matched filter & $3 \lambda / 2 D$ & 33 & 46 & 57 & 68 \\
\hline
\end{tabular}

TTFA NGS WFS option, the median magnitude of the TTFA star is 16.9 at 48 arc sec off axis, the median of the brighter of the two TT stars is at a magnitude of 19.0 at 34 arc sec off axis, and the median dimmer TT star is at a magnitude of 20.1 at 34 arc sec.

Finally, we compare the performance of the $\mathrm{SH}$ WFS quad cell with a matched filter for three TT NGS WFSs. We also investigate different pixel widths in conjunction with the matched-filter approach: $\lambda / 2 D$, $\lambda / D$, and $3 \lambda / 2 D$. The median total TT errors for the matched filter and also for the quad-cell as a comparison are displayed in Table 5. The matched-filter approach produces lower TT errors than the quad cell for all pixel sizes and read-noise levels investigated. The optimal pixel size is $\lambda / 2 D$ for 0 electrons of read noise per pixel and $\lambda / D$ for 5,10 , and 15 electrons of read noise. The improvement with the matched filter is not due to the noise term alone. Although the matched-filter approach produces less noise, this can allow for a faster sample rate to reduce wind shake and servo lag and can allow for a NGS constellation with less tilt anisoplanatism to be chosen. Using the matched-filter algorithm is contingent upon improvements in IR technology to allow for large, low noise, IR detector arrays.

\section{Conclusions}

In this paper, we have presented the modeling of sky coverage for an ELT, in particular, the TMT facility AO system NFIRAOS and have hence generated a TT error budget. From the simulations presented in Section 4 , we conclude that IR detectors are preferable to optical detectors for the NGS WFS, which is mainly due to the expected partial correction in the IR, and also to the higher stellar densities in the IR compared to the optical. We find that a 2 arc min diameter patrol field for finding NGS is sufficient.

At least one NGS WFS is required to measure the focus from the NGS in order to track variations in the sodium-layer altitude, and we find that an additional two TT NGS WFSs significantly improves the TT error for the NFIRAOS. The best TT estimate, however, is gained by using a Rayleigh LGS in conjunction with a TT NGS and the sodium LGS asterism. We have discarded this option for now, due to the optical complexity of using the LGS at two different altitudes.

Simulation results also indicate a significant improvement in using a matched-filter approach to estimating TT from the NGS rather than with a 
quad-cell Shack-Hartmann. IR detector development will be necessary to implement this option.

Although this model is largely complete, further refinements will continue to be made when more accurate telescope wind-shake and sodium-layer altitude tracking models are available and when further turbulence data is available from the proposed TMT sites.

The authors acknowledge the support of the TMT partner institutions. They are the Association of Canadian Universities for Research in Astronomy (ACURA), the Association of Universities for Research in Astronomy (AURA), the California Institute of Technology, and the University of California. This work was also supported by the Canada Foundation for Innovation; the Gordon and Betty Moore Foundation; the National Optical Astronomy Observatory, which is operated by AURA under cooperative agreement with the National Science Foundation; the Ontario Ministry of Research and Innovation; the National Research Council of Canada; and the National Science Foundation Science and Technology Center for Adaptive Optics, managed by the University of California at Santa Cruz under cooperative agreement AST-9876783.

\section{References}

1. J. Nelson and G. H. Sanders, "TMT status report," in GroundBased and Airborne Telescopes, L. M. Stepp, ed., Proc. SPIE 6267, 745-761 (2006).

2. I. Hook, G. Dalton, and R. Gilmozzi, "Scientific requirements for a European ELT," in Ground-Based and Airborne Telescopes, L. M. Stepp, ed., Proc. SPIE 6267, 726-734 (2006).

3. M. W. Johns, "The Giant Magellan Telescope (GMT)," in Ground-Based and Airborne Telescopes, L. M. Stepp, ed., Proc. SPIE 6267, 762-776 (2006).

4. T. E. Andersen, A. Ardeberg, J. Beckers, M. Browne, A. Enmark, P. Knutsson, and M. Owner-Petersen, "From Euro50 toward a European ELT," in Ground-Based and Airborne Telescopes, L. M. Stepp, ed., Proc. SPIE 6267, 716-725 (2006).

5. H. W. Babcock, "The possibility of compensating astronomical seeing," Publ. Astron. Soc. Pac. 65, 229-236 (1953).

6. D. B. Calia, B. L. Ellerbroek, and R. Ragazzoni, eds., Advancements in Adaptive Optics, Proc. SPIE 5490 (2004).

7. B. L. Ellerbroek and F. R. Rigaut, "Methods for correcting tilt anisoplanatism in laser-guide-star-based multiconjugate adaptive optics," J. Opt. Soc. Am. A 18, 2539-2547 (2001).

8. M. Le Louarn and M. Tallon, "Analysis of modes and behavior of a multiconjugate adaptive optics system," J. Opt. Soc. Am. A 19, 912-925 (2002).

9. B. Femenia, "Tip-tilt reconstruction with a single dim natural guide star in multiconjugate adaptive optics with laser guide stars," J. Opt. Soc. Am. A 22, 2719-2729 (2005).

10. D. J. Butler, R. I. Davies, H. Fews, R. M. Redfern, N. Ageorges, W. K. Hackenberg, R.-R. Rohloff, S. Rabien, T. Ott, and S. Hippler, "Sodium layer monitoring at Calar Alto by LIDAR," in Adaptive Optical Systems Technology, P. L. Wizinowich, ed., Proc. SPIE 4007, 358-367 (2000).
11. R. M. Clare and B. L. Ellerbroek, "Sky coverage estimates for adaptive optics systems from computations in Zernike space," J. Opt. Soc. Am. A 23, 418-426 (2006).

12. G. Herriot, P. Hickson, B. L. Ellerbroek, D. A. Anderson, T. Davidge, D. A. Erickson, I. P. Powell, R. Clare, L. Gilles, C. Boyer, M. Smith, L. Saddlemyer, and J.-P. Véran, "NFIRAOS: TMT narrow field near-infrared facility adaptive optics," in Advances in Adaptive Optics II, B. L. Ellerbroek and D. B. Calia, eds., Proc. SPIE 6272, 228-239 (2006).

13. R. Noll, "Zernike polynomials and atmospheric turbulence," J. Opt. Soc. Am. A 66, 207-211 (1976).

14. L. Gilles, B. L. Ellerbroek, and J.-P. Véran, "Laser guide star multi-conjugate adaptive optics performance of the Thirty Meter Telescope with elongated beacons and matched filtering," in Advances in Adaptive Optics II, B. L. Ellerbroek and D. B. Calia, eds., Proc. SPIE 6272, 1025-1032 (2006).

15. G. A. Tyler and D. L. Fried, "Image-position error associated with a quadrant detector," J. Opt. Soc. Am. 72, 804-808 (1982).

16. W. L. Melvin, "A STAP overview," IEEE Aerosp. Electron. Syst. Mag. 19, 19-35 (2004).

17. B. L. Ellerbroek and D. W. Tyler, "Adaptive optics sky coverage calculations for the Gemini-North telescope," Publ. Astron. Soc. Pac. 110, 165-185 (1998),

18. D. L. Fried, "Optical resolution through a randomly inhomogenous medium for very long and very short exposures," J. Opt. Soc. Am. A 56, 1376-1379 (1966).

19. J.-P. Véran and G. Herriot, "Woofer-tweeter tip-tilt control for NFIRAOS for TMT," in Advances in Adaptive Optics II, B. L. Ellerbroek and D. B. Calia, eds., Proc. SPIE 6272, 555-562 (2006).

20. B. L. Ellerbroek, "Linear systems modeling of adaptive optics in the spatial-frequency domain," J. Opt. Soc. Am. A 22, 310322 (2005).

21. J. N. Bahcall and R. M. Soneira, "The universe at faint magnitudes. I. Models for the galaxy and the predicted star counts," Astrophys. J. Suppl. 44, 73-110 (1980).

22. A. Spagna, "Guide star requirements for NGST: deep NIR starcounts and guide star catalogs," Space Telescope Science Institute Document NGST-R-OO13B (Space Telescope Science Institute, Baltimore, Md., 2001).

23. D. Kerley, S. Roberts, J. Dunn, N. Stretch, M. Smith, S. Sun, J. Pazder, and J. Fitzsimmons, "Validation and verification of integrated model simulations of a thirty meter telescope," in Optical Modeling and Performance Predictions II, M. A. Kahan, ed., Proc. SPIE 5867, 220-231 (2005).

24. G. Herriot, P. Hickson, B. L. Ellerbroek, J.-P. Véran, C.-Y. She, R. M. Clare, and D. Looze, "Focus errors from tracking sodium layer altitude variations with laser guide star adaptive optics for the Thirty Meter Telescope," in Advances in Adaptive Optics II, B. L. Ellerbroek and D. B. Calia, eds., Proc. SPIE 6272, 486-495 (2006)

25. J. Vernin, A. Agabi, R. Avila, M. Azouit, R. Conan, F. Martin, E. Masciadri, L. Sanchez, and A. Ziad, "1998 Gemini site testing campaign: Cerro Pachon and Cerro Tololo," Gemini Document RTP-AO-G0094 (Gemini Observatory, Hilo, Hawaii, 2000).

26. P. Puxley, "Observing constraints—sky background," Gemini Observatory, October 2002, http://www.gemini.edu/sciops/ ObsProcess/obsConstraints/ocSkyBackground.html. 